\title{
Relaciones económicas entre los Estados Unidos y América Latina
}

América Latina se ha convertido en un actor central en la eco. nomía mundial. Su dramático desarrollo durante las dos décadas pasadas, si bien dejó muchos problemas sin resolver, la ha colocado a la vanguardia del mundo en desarrollo. Este nuevo status para la parte sur del hemisferio constituye la base de las relaciones económicas actuales entre los Estados Unidos y América Latina.

Como resultado de este desarrollo, algunos palses latinoamericanos, particularmente Brasil, Mexico y Venezuela, juegan ahora un rol importante y creativo en el comercio y las finanzas internacionales. Por lo tanto, las relaciones económicas son cruciales para la totalidad de la política estadounidense hacia la región -y la región figura en forma crucial dentro del conjunto de la polftica económica internacional de los Estades Unidos.

Sin embargo, los Estados Unidos no poseen una "politica económica" con respecto a América Latina. Más bien, nuestra política económica global pretende tomar en consideración las necesidades y preocupaciones de América Latina, y consultar con estas naciones toda la gama de los temas económicos internacionales. Después de revisar la impresionante envergadura y profundidad del desarrollo latinoamericano, y lo que este significa para los Estados Unidos, describiré las polfticas actuales y añadiré algunas ideas acerca de posibles directivas para las relaciones hemisféricas a medida que vayan ocurriendo nuevos cambios en el status de la región y en el ambiente económico global.

\section{DESARROLIO LATINOAMERTCANO}

América Latina ha sobrepasado a todas las otras naciones en desarrollo en su ritmo de progreso económico.

Entre 1967 y 1977, el producto nacional bruto de la región aumentó en más del doble en términos reales, casi hasta llegar a los US $\$ 400$ mil millones. Esto representa una tasa anual de crecimiento del 6,1 por ciento, comparada con el 5,1 por ciento de los palses en desarrollo y un 3,9 por ciento de los paises desarrollados.

Durante los años 1973-1977, la región creció en una tasa prome- 
dio anual de casi un 5 por ciento para los países de la $\mathrm{OECD}$. Inclusive, la región mantuvo un crecimiento impresionante durante el proceso recesivo mundial, mitigando el impacto de la recesión en los paises industrializados, particularmente en los Estados Unidos.

El PNB real per capita en la región ha aumentado en más de la mitad desde 1965 . Permanece ahora en us $\$ 1.100$, comparado con un PNB per cápita de Us $\$ 450$ para el resto de los países en desarrollo.

Este rápıdo crecimiento económico y el nivel de desarrolio rclativamente alto, covirtieron a América Latina realmente cu parte de una suerte de nueva "clase media internacional", junto con unos pocos países en el Medio Oriente y el Sudeste asiático. América Latma no está, de ninguna manera, totalmente desarrollada y, de hecho, subsisten grandes bolsones de pobreza, inclusive dentro de los paíscs más avanzados del hemisferio. Llaramente,no: otros reconocemos y rispetamos la diversidad y la individualidad de las naciones de la región. Pcro la región como un todo goza de standards de vida nilucho más altos que aquellos que predominan en los paises en desarollo de Africa y Asia, y ha llegado a ser un factor importante en los mercados comerciales y financieros claves a lo largo de todo el mundo.

$\mathrm{El}$ progreso de la región ha conlundido, si es que no ha borrado, la distinción tradicional cntre los países desarrollados y monos desarrollados. En realidad, hemos acuñado un nuevo término -paises en desarrollo más adelantados (ADC*) - para caraclicizit cstos (y atgunos otros) poderes económicos emergentes. Como consecuencia ds su progreso, vemos a América Latina como un instrumento que permitirá acuñar nuevas formas de cooperación internacional, proporcionando un progreso significativo en las relaciones Norte-Sur -en vez de una retórica imprudente- con beneficios reales para todos los participantes, vengan ellos del Norte o del Sur del Río Grande.

\section{LA POLITICA ECONOMICA INTERNAGIONAL DE LOS ESTADOS UNIDOS}

La política económica internacional de los Estados Unidos posee dos elementos cardinales: el apoyo a nuestros objetivos económicos nacionales, tales como el pleno empleo y la estabilidad de precios, y el mantenimiento de un efectivo sistema económico internacional. Ninguno de ellos puede prosperar permaneciendo aislado del otro, ni tampoco pueden prosperar sin contar con un compromiso total por parte de los ADC.

Por lo tanto, apoyamos una plena participación por parte de to dos los países, incluídos los ADC, en las decisiones que los afectan

* En adelante se conservará la sigla correspondiente a la expresión original en inglés, advanced developing countries. 
tanto a eilos como al funcionamiento del sistema económico mun. dial. Tal participación, por supuesto, requiere la aceptación de su responsabilidad por parte de cada pais, en concordancia con su estado de desarrollo.

Estcs dos elementos son inseparables. En la medida que los paises acepten mayores responsabilidades en el sistema económico internacional, su presencia en el sistema y su habilidad para influir en su desarrollo debe aumentar proporcionalmente. Los ADC recibirán, sin duda, un gran beneficio de este mayor papel, puesto que serán capaces de conseguir que sus intereses sean contemplados en forma adecuada en el desarrollo del sistema económico internacional, asl como la colaboración necesaria para asegurar el fortalecimiento de dicho sistema en si mísmo, lo que es crucial para sus necesidades nacionales.

Me gustaria describir brexemente cómo nuestras politioas llevan a cabo esos principios en las arenas más importantes de nuestra interacción económica con la región: comercio, desarrollo financiero y el sistema monetario internacional. No me referire a otros puntos tan relevantes como los productos básicos, la energía y la polftica de inversión, para poder concentrarme en aquellos que mejor ilustran el nuevo modelo de relaciones hemisféricas.

\section{COMERCIO}

Es probable que el comercio sea el área principal de interacción económica de los Estados Unidos con América Latina, puesto que los países de la región que se están industrializando con rapidez necesitan ante todo el acceso a los mercados de los paises desarrollados. Nuestro enfoque presenta aqui dos aspectos: el rechazo de muchas propuestas para restringir las actuales importaciones estadounidenses de América Latina, y las Negociaciones Comerciales Multilaterales, en donde estamos trabajando en forma muy activa para reducir las barreras tarifarias y no tarifarias al comercio por parte de todas las naciones del mundo y para mejorar las regulaciones que gobieman los flujos del comercio internacional. En particulat, nuestras propuestas en el área de las tarifas reflejan nuestro compromiso para aumentar el acceso de los productos latinoamericanos en el mercado de los Estados Unidos.

También esperamos que estas naciones cumplan con su parte al ayudar a mejorar el sistema de comercio global, en consistencia con sus propias situaciones en materia de comercio y desarrollo. Esto significa una creciente aceptación, por parte de los $A D c_{\text {, }}$ de por lo menos una reciprocidad parcial en 'las Negociaciones Comerciales Multilaterales: por ejemplo, un mayor compromiso para limitar las prácticas gubernamentales en materia de adquisiciones que discri- 
minan en contra de los proveedores extranjeros, y su adhesión al Convenio Internacional sobre Créditos de Exportación. En general, esto significa una renuncia progresiva a los tratamientos especiales a medida que los páses avanzan en el proceso de desarrollo, de manera que los países más necesitados puedan beneficiarse más plenamente de las preferencias existentes.

La aceptación de mayores responsabilidades en las relaciones comerciales es especialmente importante en cuanto al uso de subsidios gubernamentales. Uno de los objetivos de las Negociaciones Comerciales Multilaterales es alcanzar un acuerdo sobre los subsidios y los derechos compensatorios.

Necesitamos poner un tope al creciente uso de subsidios para incentivar el crecimiento orientado hacia las exportaciones a expensas de otras naciones competidoras.

- Necesitamos ampliar y profundizar el compromiso, que fue aceptado previamente por la mayoría de las naciones industrializadas, para no usar los subsidios de exportación.

-Necesitamos una nueva disciplina internacional para prevenirnos contra la protección disfrazada de los mercados domésticos a través de los subsidios internos o a la producción.

- Necesitamos fortalecer las disposiciones actuales del GATT sobre solución de conflictos, para asegurar que estas reglas sean cumplidas en forma efectiva.

Es evidente que los subsidios pueden jugar un papel importante en la polf́tica económica nacional, y que se necesita flexibilidad en las reglas aplicables a países que están situados en diferentes peldaños de la escala del desarrollo. Los países totalmente desarrollados deberían aprobar inmediatamente todas las medidas del acuerdo, en tanto que habría que acordar un tratamiento especial y diferencial para los países en desarrollo. Sin embargo, dicho código debería proveer lo necesario para que, a medida que las industrias de los ADC lleguen a ser internacionalmente competitivas, éstos aumenten cada vez más sus obligaciones. También este código debe conseguir que se acepte desde el comienzo el principio de que sus subsidios no deberían perjudicar a otros países. Reconocemos plenamente la naturaleza evolutiva de este proceso, y por ello aceptamos que estas obligaciones puedan ser cumplidas en diversas fascs, y no que tengan que ser instituídas de una sola vez.

Hemos estado trabajando sobre este problema en estrecho contacto con las naciones en desarrollo, especialmente con el Brasil. Este país merece un gran crédito por haber elaborado medidas a través de los cuales tal código puede aplicarse a los países en desarrollo, de manera que defienda sus legitimos intereses nacionales y al mismo tiempo fortalezca el sistema comercial global. El lide- 
razgo y creatividad del Brasil ya han jugado un papel central en las Negociaciones Comerciales Multilaterales, y sin duda continuarán haciéndolo. Otro ejemplo de beneficio mutuo es el acuerdo a que se llegó con el Uruguay, para que los Estados Unidos renuncien a los derechos compensatorios en los productos del cuero y del calzado, el Uruguay acepte eliminar totalmente sus sustanciales subsidios de exportación, comenzando por los productos del cuero para principios de 1979 , e incluyendo los demás productos a partír de 1983.

Nuestra facultad para renunciar a la aplicación de los derechos compensatorios tiene como plazo de expiración el $\$$ de enero de 1979. La pérdida de esta facultad, y la imposición de derechos contra las exportaciones de algunos denuestros principales socios comerciales podria muy bien perturbar profundamente las Negociaciones Comerciales Multilaterales, Ello comprometerla el notable progreso que ya se ha alcanzado con diversos paises latinoamerica. nos hacia la resolución definitiva de los problemas derivados de sus subsidios de exportación, junto con el progreso - muy publicitado- que hemos hecho con la Comunidad Económica Europea. La extensión del plazo para usar la facultad de renunciar a estos derechos, dependiente de la conclusión de un satisfactorio paquete de acuerdos en el marco de las Negociaciones Comerciales Multilaterales antes del 2 de enero de 1979, incluyendo un código sobre derechos compensatorios y subsidios, es por lo tanto crítico, no sólo en términos de todos los intereses económicos de los Estados Unidos, sino también de nuestras relaciones con América Latina y de una continuada y afectiva colaboración hemisferica en materias comerciales

Por supuesto, un gran volumen de nuestro comercio con América Latina ya dispone de un acceso libre de impuestos al mercado de los Estados Unidos bajo el actual sistema de tarifas y el sistema generalizado de preferencias (SGP). El valor total de las importa. ciones libres de impuesto realizadas dentro del scp desde América Latina en los seis primeros meses de 1978 fue de us $\$ 16$ millones, sobre un total de us\$2.400 millones para todas las naciones en desarrollo. Las importaciones libres de impuesto en virtud del scP, provenientes de todos los paises de menor desarrollo, aumento en la impresionante proporción de $\$ 1 \%$ entre enero y junio de 1978 , en comparación con el mismo periodo en 1977. Los palses de América Latina, particularmente Argentina $(91 \%$ ) y Brasil (más de $56 \%$ ), hicieron grandes progresas.

En esto tambien nuestra politica aplica un principio gradualista. Cuando un deterninado producto de un pats elegible para el sGP llega a ser competitivo en el mercado estadounidense, ese producto 
retrocede a un tratamiento tarifario normal, sobre la base de que ya no se necesita de una ayuda especial y de que el no haccrlo impediría en forma injusta que países menos competitivos tengan la oportunidad de entrar a ese mercado. Cientoveintidós productos calificaron para este tratamiento gradual en 1978, de los cuales 79 eran de Brasil y México solos.

\section{DESARROLLO FINANGIERO}

Nuestra política global en el área del desarrollo financicro consiste en asegurarnos de que los países pobres reciban los recursos adccuados, en términos apropiados, que los ayuden en sus esfuerzos para reducir la extrema pobreza y alcanzar un crecimiento autosostenido. La aplicación de esta política global para América Latina significa que la región, a causa de su progreso en el desarrollo, debería trasladarse gradual pero deliberadamente desde 1) la ayuda concesional, como la proporcionada por el aid y los préstamos blandos de los Boncos Multilaterales de Desarrollo (BDM), hacia 2) los préstamos no concesionales de estas últimas instituciones y de los mercados privados de capital y 3) hacia posiciones que les permitan ayudar a sus vecinos más pobres a través de diversos canales de ayuda, bilaterales y multilaterales.

Gran parte de este cambio ya se ha realizado en América Latina. Solo seis pequeños países en la región son actualmente elegibles para préstamos del IDA, la institución afiliada al Banco Mundial que proporciona préstamos blandos. Durante los últimos años, los Estados Unidos han decidido terminar con los programas de aID en Argentina, Brasil, Colombia, Chile, Ecuador, Uruguay y Venezuela; el nivel de las donaciones y préstamos de la AID para la región declinó en un promedio de más de ss\$ 600 millones por año durante mediados de los años 60 hasta menos de us\$ 250 millones durante el último año fiscal, cuando el énfasis de nuestra ayuda bilateral se trasladó hacia las regiones nás pobres de Africa y el Sur de Asia. Venezuela y Trinidad Tobago ya no piden préstamos al Banco Mundial. Los países más avanzados han obtenido un amplio acceso a los mercados privados de capital. Unos pocos países en la región han comenzado a montar sus propios mecanismos de asistencia externa para ayudar a los países más pobres de entre los de menor desarrollo relativo.

Nuestra actitud frente al Banco Interamericano de Desarrollo tal vez refleja mejor estos principios. Nuestro rol en el Banco, y aquel de los países latinoamericanos, ha ido evolucionando gradualment? en respuesta al progreso de desarrollo de la región. Hemos alentado a los países avanzados de la región a proporcionar recursos concesionales a sus vecinos m.enos afortunados, ante todo refrenándose 
de pedir préstamos blandos al BID (del Fondo de Operaciones Especiales) y luego para que aumenten la cantidad de recursos convertibles con que ellos contribuyen a dicho Fondo. En la última operación de reposición de recursos, Argentina, Brasil y Mexico reuunciamon al uso de los recursos convertibles del Fondo de Operaciones Especiales y aportaron un cuarto de su contribución al mismo en monedas convertibles.

Estamos ahora negociando la quinta Reposición de Recursos con el aID, a fin de cubrir las operaciones del Banco para los años 1979 . 82. Tenemos confianza en que los resultados lograrán que se cumplan nuestros objetivos de diversas maneras, y que proporcionarán un indicio más de la madurez de la región y de nuestra relación con ella:

- Un acuerdo para negar el acceso al Fondo de Operaciones Especiales a otros palses que ya no necesitan ayuda concesional;

-Un Fondo de Operaciones Especiales más pequeño en total, como convenga al desarrollo de la región en general;

-Una creciente participación de la contribución de los principales paises de la región al Fondo de Operaciones Especiales en mo. neda convertible, indicando sus crecientes fuerzas fiñancieras y económicas;

- Un acuerdo para concentrar el grueso financiamiento concecional en los países más pobres, y para canalizar e incrementar una patricipación creciente de los recursos del nid hacia proyectos destinados a alcanzar a los pobres; y

- Una limitación al uso de los recursos de capital del sto por parte de los palses de mayor desarrollo del hemisferio, poniendo una cuota mayor a disposición de los países más necesitados, que no tienen tanto acceso a los mercados de capital privado. La extensión de los préstamos a los palses de mayor desarrollo será determinada en gran medida por nuestra contribución al capital del Banco, que dependerá de la buena voluntad de estos países de mayor desarrollo para proporcionar ellos mismos los recursos adicionales para ayudar a los palses más pobres de la región.

\section{TEMAS MONETARIOS}

Ia polftica monetaria internacional de los Estados Unidos ha estado dirigida a asegurar el continuado y efectivo funcionamiento de un sistema mundial, abierto de comercio y de pagos, del cual todos los paises se benefician notablemente, incluyendo aquellos de América Latina. Se están tomando una cantidad de medidas especificas con este objeto en el Fondo Monetario Internacional (FMI), las que deberfan contribuir tanto directa como inderectamente a la consecusión de las metas económicas de América Latina. 
Recientemente se alcanzó un consenso acerca de mediclas impor. tantes para fortalecer el FMI. Una consiste en un 50\% de aumento en las cuotas clel FMI, sobre lo cual se tomará una decisión al terminar este año. Un aumento así incrementaría los recursos permanentes del rar en aproximadamente 25 mil millones, con un aumento cquivalentc en el porcentajc de acceso de todos los países miembros, incluyendo aquellos de América Latina, al financiamiento del Fondo.

Este aumento de las cuotas va a asegurar que el FMI sea capaz de continuar satisfaciendo la necesidad que tiene la economía internacional de contar con financiamiento "condicional" de las balanzas de pages, por ejemplo, suministrar financiamicnto sujeto al requerimiento de que el país que obtiene el préstamo implemente mediclas apropiadas de políticas con el objeto cle superar sus problemas de pagos. Muchos de los países latinoamericanos han pecliclo préstamos al FMi en los últimos años, y han experimentado la consiguiente mejora en su situación económici. El Fm está ahora trabajando en forma estrecha con varias naciones de América Litina, con el fin de ayudarlas a superar sus problemas de pagos.

Un segundo consenso, que también se concretará dentro de 1978, consiste en que el FMI debcría distribuir anualmente 4 mil millons de Derechos Especiales de Giro (DEG) equivalentes a alredeclor de 5 mil millones de dólares durante los próximos tres años. Tales aportes, que incluirán la distribución de los Deg recientemente creados a sus países miembros de acuerdos con sus cuotas, han sido procunados desde hace mucho tiempo por los miembros latinoamericanos del FMr. Ellos ayudarán a satisfacer la necesidad glolbal de largo plazo de suplementar las reservas existentes en una mancra que contribuya a la estabilidad del sistema monetario.

Tercero, cl inminente establecimiento de un fondo financiero suplementario - la así llamada "Witteveen Facility"- que va a fortalecer en forma significativa la capacidad del FM para ayudar a aquellos de sus miembros que estén experimentando problemas particularmente serios de balanza de pagos. Este fondo de $10,8 \mathrm{mil}$ millones de dólares va a suplementar temporalmente los recursos del FMI durante un período de gran tensión en el sisicma monctario. Estará disponible para todos los miembros que satisfagan los criterios para girar contra este fondo, incluyendo a los países en desarrollo. Esto se opone al Fondo de Ayuda de la oEcD, que fue una proposición del gobicrno anterior, y que habria estado solamente a disposición de los países industrializados. Me alegra el que la legislación que autorizó la participación de los Estados Unidos en este Fondo haya sido aprobada por el Congreso, y espero que pronto sea aprobado el aporte de la cuota requerida.

El concenso a que se ha llegado para expandir los recursos del 
Fu ha sido alcanzado con la total participación de los paises en desartollo, incluidas las naciones latinoamericanas. En consistencia con nuestra politica de apoyar una participación con responsabilidad, los paises latinoamericanos juegan un rol importante en el proceso de toma de decisiones del rmi. Tres de los veinte miembros tanto del Comité Interino como el Consejo Ejecutivo del Fan son latinoamericanos. Las cuotas y las votaciones de varios miambros latinoamericanos, incluidos Venezuela, Brasil y México, fueron aumentadas a príncipios de este año. Veneruela y Guatemala se laan comprometido para proporcionar aproximadamente 640 millones $y$ 38 millones de dólares respectivamente al fondo financiero suplementario. En esto también trabajamos unidos con América Latina, como socios en el manejo económico global.

\section{DIRECTVAS YUTURAS}

A medida que avanza el proceso de dearrollo en América Latina, cosa que creemos continuará sucediendo, podemos pensir en la evolución futura de las relaciones entre los Estados Unidos y Amé. rica Latina.

Creemos que la región deberia avanzar hacia un sistema más abierto en sus relaciones comerciales y financieras con el resto del mundo. Hay tendencias positivas dirigidas hacia esto, pero también existe el peligro de que algunos parses se resistan a una apcrtura. La interdependencia de las politicas se vuelva crucial en este punto: los Estados Unidos debemos mantener abiertos nuestros merca. dos para los productos y los créditos de América Latina si esperamos que ellos abran sus economias, y viceversa.

En la medida en que el Norte o el Sur de América continúen intentando liberalizar sus relaciones económicas con el resto del mundo, se tornarản necesarias y deseables nuevas formas de cooperación adicionales.

-En el criticamente importante campo comercial, la meta más urgente parece ser a plena participación y asociación con el GATr. Greemos que todos los paises latinoameticanos deberian formar parte del CATT y participar lo más plenamente posible en las negocia. ciones comerciales multilaterales.

-La liberalización comercial que esto implicará deberia aumen. tar la interdependencia entre América Latina y el resto de la economia mundial. Esto, a st vez acentuará la necesidad de mantener un intercambio de conszllas e informaciones sobre las tendencins de corto plazo de la economia mundial. Deberfamos pensar cómo podria llevarse mejor a cabo este proceso.

- Creemos que las posibilidades que hay en el campo de la inversión son particularmente interesantes. A medida que van des- 
gastándose las antiguas idcologias sobre la inversión extranjera, que ha dado lugar a muy diferentes puntos de vista sobre la materia, vemos mucho mayores oportunidades para la cooperación. Los países avanzados de la región comprenden plenamente los benelicios que se derivarían, tanto para los países anfitriones como para los países sedc, si se asegurara que las corporacioncs multinacionales jugarán un rol constructivo en la economía mundial, y scrán capaces de negociar en forma efectiva con estas firmas para consoguir sus propios objetivos nacionales. Esta nueva situación nos puede permitir acercarnos al logro de acuerdos sobre las "reglas de juego" que deben regir la inversión internacional.

- En el área de la ayuda, buscamos la creciente colaboración con los países de mayor desarrollo relativo en el mancjo de las insticuciones multinacionales de crélito y otros mecanismos de tranferencia de recursos.

Está claro que el Congreso de los Estados Unidos tienc un papel crucial en cada uno de estos temas: comercio, financiamiento del desarrollo, temas monetarios, inversión, productos básicos y muchos otros. Por lo tanto, damos una gran bienvenida a estas sasioncs y esperamos poder continuar trabajando estrechamente con el Congreso para elaborar e implementar diversos aspectos de la política cconómica de los Estados Unidos hacia América Latina.

\section{CONCLUSION}

Ll rol de los paises latinoamericanos, en especial de aquellos de mayor desarrollo, pasa a través del espectro total de las relaciones eco. nómicas estadounidenses.

-Como poderes económicos intermedios y en rápido crecimiento, creemos que deben asumir mayores responsabilidades.

-Como receptores de mayores beneficios provenientes del co. mcrcio nundial, sus prácticas comerciales deberían conformarse citda vez más con las reglas que se aplican a los principales protagonistas de la economía internacional.

-Aquellos países deberían depender menos de la ayuda concesional bilateral y multilateral, de manera que un volumen mayor de recursos esté a la disposición de sus vecinos menos afortunados.

-En suma, ellos deben trabajar más estrechamente con los países inclustrializados para proporcionar un liclerazgo en la cconomía mundial.

Esta crecicnte responsabilidad tracrá mảs beneficios a lat región. Un mayor compromiso en el manejo del sistema económico internacional por parte de los países de la región les asegurará una voz más potente en su desarrollo futuro, haciéndolos menos dependien- 
tes de las decisiones tomadas por otros y más capaces de determinar la cvolución de sus relaciones económicas con el resto del mundo.

En esencia, la participación total de América Latina en la economía global servirá para prevenir que otros países adopten decisiones que no tomen plenamente en cuenta los intereses latinoamericanos. Y a causa de las ganancias que tanto América Latina como nosotros obtendríamos de un sistema económico internacional mís liberal, ambas partes se beneficiarán de un proceso de participación y responsabilidad compartida. 